\title{
Denoising of EEG Signal using Matlab and SIMULINK Techniques and Estimation of Power Spectral Density of EEG Signal using SIMULINK AR Models
}

\author{
B.Krishna Kumar
}

\begin{abstract}
The Electroencephalogram (EEG) is the standard technique for investigating the brain's electrical activity in different psychological and pathological states. Analysis of Electroencephalogram (EEG) signal is a challenging task by reason of the presence of different artifacts such as Ocular Artifacts (OA) and Electromyogram. Normally EEG signals falls in the frequency range of DC to $60 \mathrm{~Hz}$ and amplitude of 1-5 $\mu \mathrm{v}$. Ocular artifacts do have the similar statistical properties of EEG signals, often interfere with EEG signal, thereby making the analysis of EEG signals more complex. In this research paper, removal of artifacts was done using wavelets (matlab coding) as well as using SIMULINK DWT and IDWT blocks and estimated the SNR. In the next stage the output of IDWT block was taken as input to Burg model and Yule walker model to estimate the power spectral density of EEG signal by setting the various parameters of the blocks. The implementation of denoising of EEG signal using SIMULINK DWT and IDWT blocks and estimation of power spectral density of denoised EEG signal using Burg model and Yule walker model was explained in detail in the paper under the methodology heading. In this research paper, the collected EEG signal is normalized and later linearly mixed with the normalized EOG signal resulting in a noisy EEG signal. This noisy EEG signal is decomposed to 4 levels by using different wavelets. This decomposition of EEG signals yields approximate and detail coefficients. Later different thresholding techniques were applied to detail coefficients and estimated the Signal to Noise Ratio of it and estimated the power spectral density of denoised EEG signal obtained from dB4 wavelet as it is providing better SNR than other wavelets mentioned in the results.

Keywords-WT, DWT, Ocular Artifacts, power spectral density
\end{abstract}

\section{INTRODUCTION}

Electroencephalogram (EEG) is a standard non-invasive technique with which one can record the brain's electrical activity over a period of time, using electrodes. This is very much useful in diagnosing the disorders of human brain such as epilepsy, seizures and brain damage. [1-2]. EEG has been

Revised Manuscript Received on December 30, 2019.

* Correspondence Author

Dr. B. Krishna Kumar*, Professor, Electronics and Communication Engineering, Methodist College of Engineering and Technology, Hyderabad, India.

(c) The Authors. Published by Blue Eyes Intelligence Engineering and Sciences Publication (BEIESP). This is an open access article under the CC BY-NC-ND license (http://creativecommons.org/licenses/by-nc-nd/4.0/) performing a vital role to investigate brain activities in clinical application and scientific research for several years [3-5]. The EEG signals can be contaminated by various artifacts, of which the major noise source is ocular artifact. Eye-movements and eye-blinks are the major sources of ocular artifacts [6]. However, artifacts are the major enemies of high-class EEG signals. The mixing up of these ocular artifacts with the EEG signal at the time of recording causes the problems in the precise estimation of EEG signal. These artifacts will plunge into either of the 2 categories namely, technical and physiological artifacts. Power line noise $50 / 60 \mathrm{~Hz}$ falls into technical artifact category while the artifacts that crop up because of ocular(EOG), heart(ECG) and muscular activity(EMG) falls into physiological artifacts category respectively [7].

Regression in the time and frequency domains [8-10] methods were implemented in removing eye blinks artifacts. These methods require a reliable reference channel. This channel can be contaminated by EEG. So, EEG has to be removed from the reference channel by regression techniques. Hence, the regression methods are not the finest to remove EOG artifacts.

In this research paper, noisy EEG signal was decomposed to four levels using different wavelets. This decomposition gives low frequency and high frequency components of noisy Electroencephalogram signal. The high frequency components contain more noise information than low frequency components, hence are processed with various thresholding techniques.

The above mentioned procedure has been carried out both in matlab and SIMULINK.

\section{METHODOLOGY}

\section{A) Wavelet Denoising and Simulink denoising}

EEG signal that is collected from Physionet data base[11] is normalized by using the following formula:

Normalized EEG signal =

collected EEG Signal - Mean(Collected EEG Signal)

Std (Collected EEG Signal)

The collected and normalized EEG signals are shown in Figure.1. 


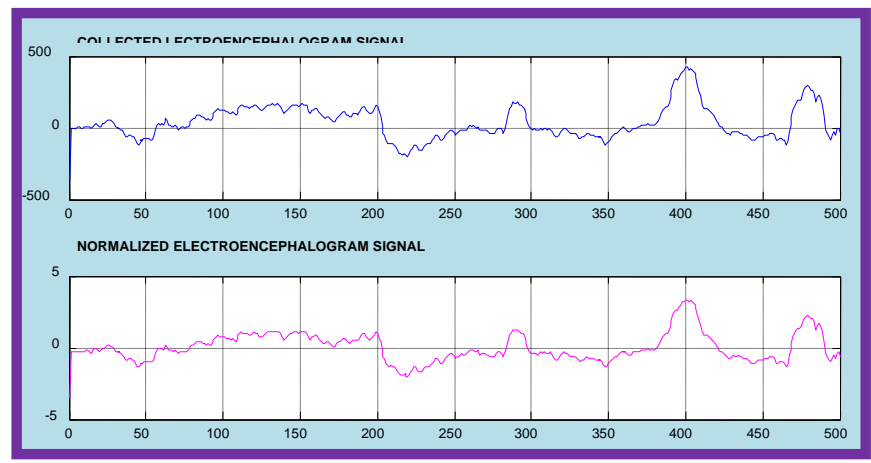

Fig 1: Collected EEG Signal and the Normalized EEG Signal

The normalized EEG signal from matlab workspace is taken to SIMULINK, using signal from work space block and the output of this block is connected to scope to view the signal and the normalized EEG signal from the scope block of SIMULINK is shown in the Fig.2.

For convenience sake, normalized signal both from matlab and as well as SIMULINK are shown in Fig.2

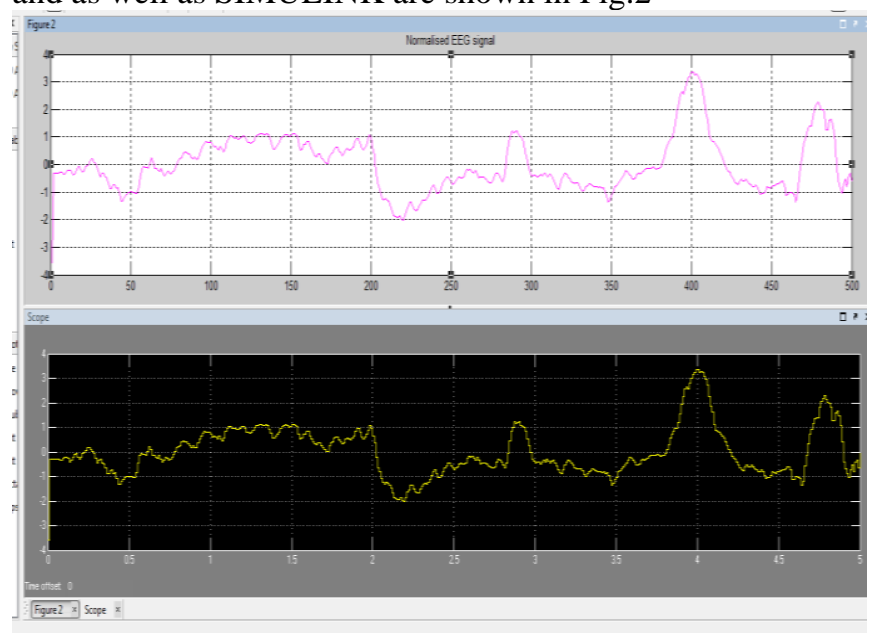

Fig 2: Normalized EEG Signal using matlab and SIMULINK

The EOG signal that is collected from Physionet data base is normalized by using the following formula:

Normalized EOG signal =

Collected EOG Signal - Mean (Collected EOG Signal) Std (Collected EOG Signal)

The collected and normalized EOG signals are shown in Figure 3.

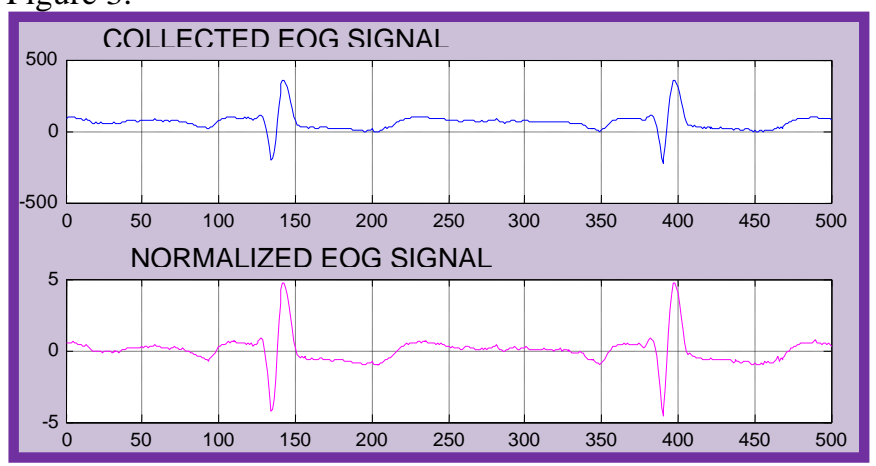

Fig 3: Collected ElectroOculoGram Signal and Normalised EOG Signal

The normalized EOG signal from matlab workspace is taken to SIMULINK, using signal from work space block and the output of this block is connected to scope to view the signal. The normalized EOG signal from the scope block of SIMULINK is shown in the Fig.4.
For convenience sake, the normalized signal both from matlab and SIMULINK are shown in Fig.4.



Fig 4: Normalized EOG Signal using matlab and SIMULINK

The corrupted EEG (observed) signal can be modeled in the following manner:

$\mathrm{y}(\mathrm{n})=\mathrm{x}(\mathrm{n})+\sigma \mathrm{e}(\mathrm{n})$

Where, $x(n)$ is the original Electroencephalogram signal ,e(n) is the ElectroOculoGram signal, $\sigma$ is the noise variance and $\mathrm{y}(\mathrm{n})$ is the Noisy EEG signal.

To achieve the noisy EEG signal, the normalised Electroencephalogram signal is mixed with the ElectroOculoGram signal with noise variance of 0.4 , [12] and is shown in the Figure 5.

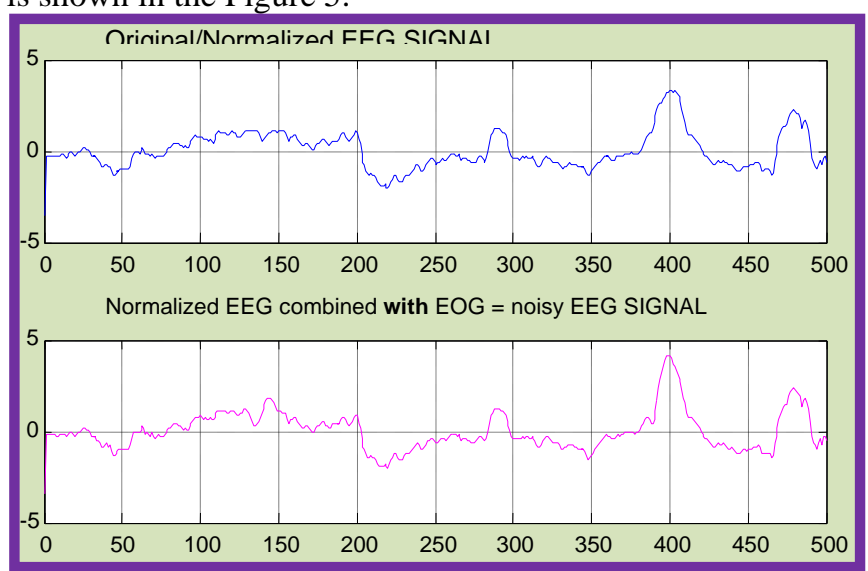

Fig 5: Normalized EEG Combined with EOG- Noisy EEG

The normalized EEG signal combined with EOG from matlab workspace is taken to SIMULINK using signal from work space block and the output of this block is connected to scope to view the signal and the normalized EEG signal combined with EOG signal from the scope block of SIMULINK is shown in the Fig.6.

For convenience sake, both the normalized EEG signal combined with EOG signal from matlab and SIMULINK are shown in Fig.6. 


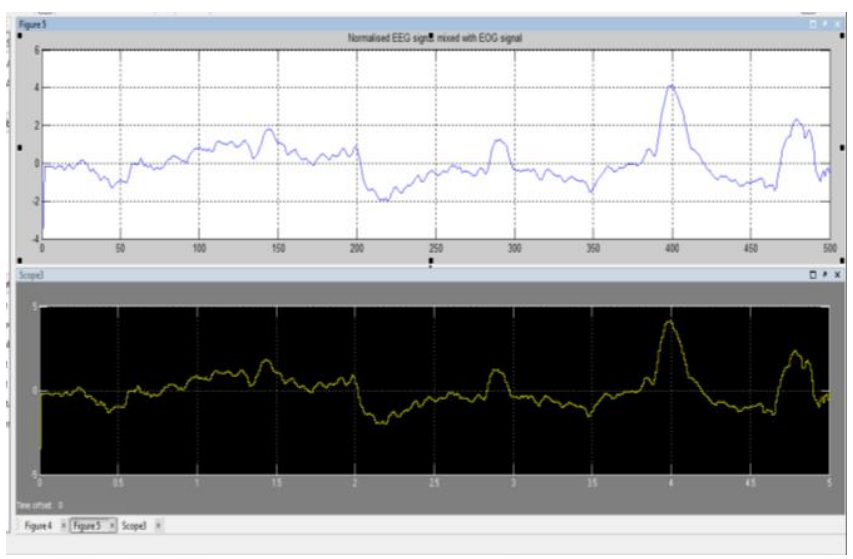

Fig 6: Normalized EEG Combined with EOG- Noisy EEG using MATLAB and SIMULINK

The decomposition of noisy EEG signal is done to four levels by dB4 wavelet. This decomposition gives Approximate and Detail coefficients of noisy Electroencephalogram signal. Soft Thresholding is applied to Detail coefficients. The wave reconstruction was done using threshold coefficients and approximate coefficients and is shown in Fig 7.

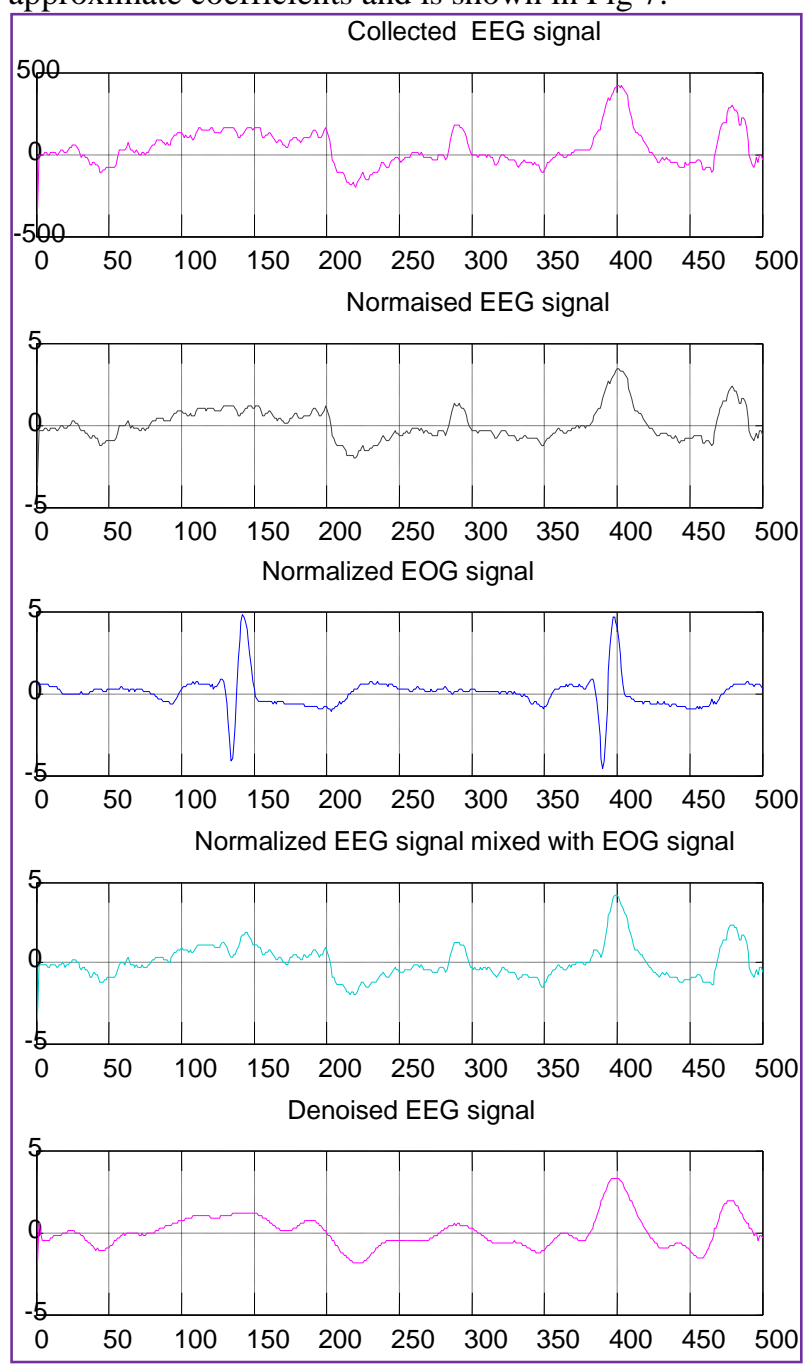

Fig 7: COLLECTED, NOISY and DENOISED EEG Signal Using Soft Thresholding Using dB-4 Wavelet

The overall set up of denoising of EEG signal using SIMULINK is shown Fig 8.

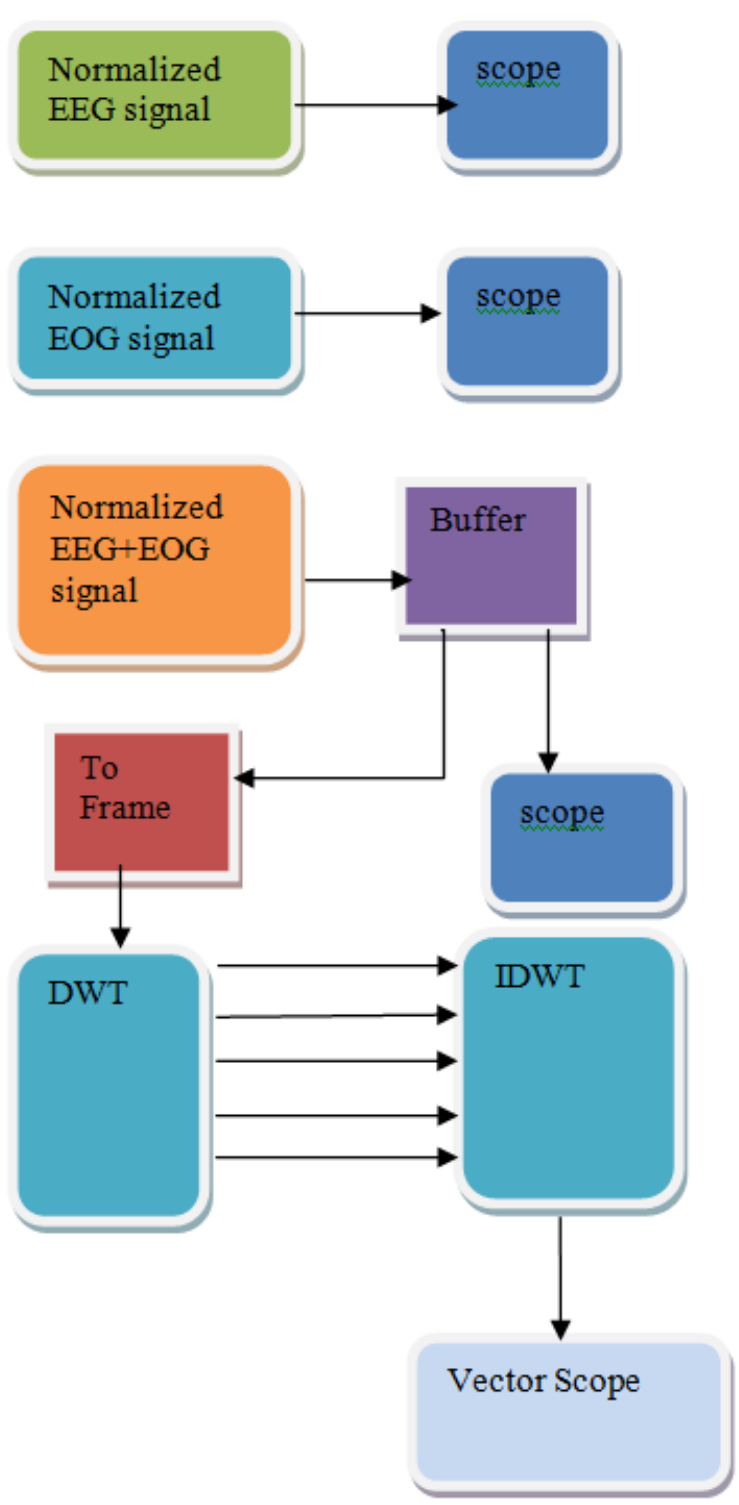

Fig 8: Set up of SIMULINK Model

The noisy EEG signal consisting of 500 samples is provided to a buffer block. Using this Buffer block these samples are converted into frames and the output buffer size per channel is considered as 16.The samples in the form of frames are provided to DWT block [13]. The DWT block is the same as that of Dyadic Analysis Filter Bank block. Basically this block computes the discrete wavelet transform (DWT). DWT block consists of a filter bank with specified high pass and low pass FIR filters. The FIR filters are used to decompose the input into subbands that have smaller bandwidths and slower sample rates. The filters can be user-defined or wavelet-based. In general, the high pass and low pass filters should be half-band filters designed to complement each other.The output of DWT block can be either taken to a single port or to multiple ports. When this output parameter is set to 'Multiple ports' the block outputs each subband from a different port as a frame-based vector or matrix. The topmost port outputs the subband with the highest frequency band. When 'Output' is set to 'Single port' the block outputs one sample-based vector or matrix of concatenated subbands.

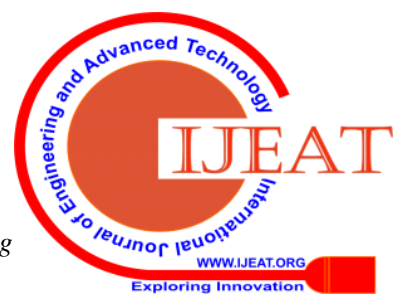


For a DWT block, the Inputs must be frame-based with a frame size a multiple of $2^{\wedge} n$, where $n$ is the value entered in the 'Number of levels.' To create a filter bank that accepts sample-based inputs and inputs of other sizes, use Two-Channel Analysis Subband Filter blocks

The parameters of this block are set in the following manner: The type of wavelet chosen was Daubechies and wavelet order is 4 . The number of levels of decomposition was set to 4 , the type of tree was chosen as asymmetric and the output of DWT block is taken to multiple ports. Since the number of levels of decomposition is taken as 4 , then the frame size becomes 16 and the total number of frames will be 32 (500/16). The samples are thresholded using ode 45 solver and later provided as input to IDWT block for the reconstruction of the signal. When 'Input' to this block is set as 'Multiple ports', one must input each subband through a different input port as a frame-based vector or matrix (the topmost input port should receive the subband with the highest frequency band).The parameters of this block were set as same as DWT block. The output of IDWT block is given to vector scope to view the denoised signal and is shown in the Fig 9.

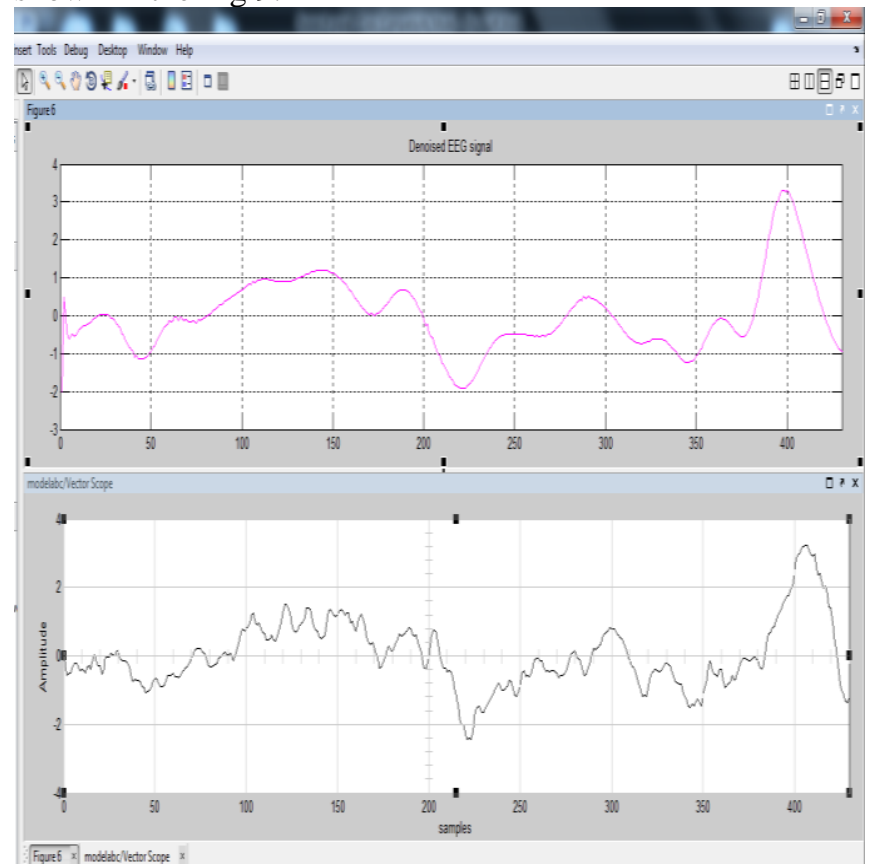

Fig 9: Denoised signal using MATLAB and SIMULINK

\section{B) BURG Method}

In this the power spectral density of EEG signal is estimated using BURG method. The Burg Method block estimates the power spectral density (PSD) of the input frame(s). The estimation order of BURG is taken as 15.This method fits an Auto Regressive (AR) model to the signal by minimizing (least squares) the forward and backward prediction errors. The input to this block is frame-based vector (column only) and size of each frame is taken as 16 . This input represents frames of consecutive time samples from a single-channel signal. Since the number of samples considered as 16 per frame and the number of frames for single channel signal of 500 samples will be 32. The output of BURG method block provides a column vector containing the estimate of the power spectral density of the signal at $N_{f f t}$ equally spaced frequency points. The frequency points are in the range $[0$, $F s$ ), where $F_{s}$ is the sampling frequency of the signal [13]. The sampling frequency of the EEG signal considered for this research work is $100 \mathrm{~Hz}$. The power spectral density obtained from BURG method is shown in the Figure 10.

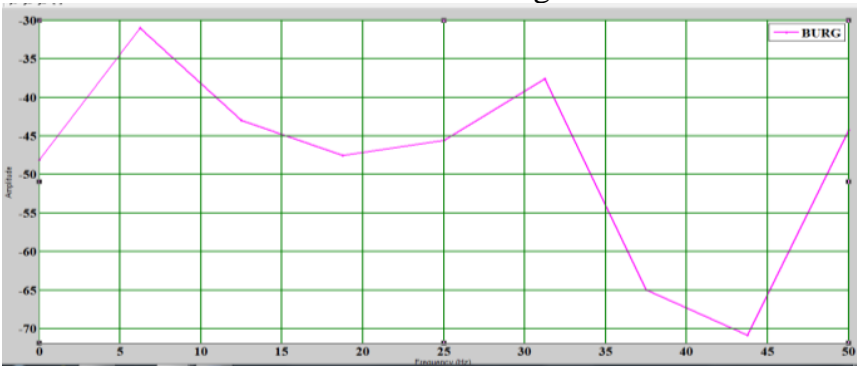

Fig 10: Power Spectral Density from BURG Method

\section{c) Yule Walker Method}

The Yule-Walker Method block estimates the power spectral density (PSD) of the input using the Yule-Walker AR method. The estimation order of Yule Walker Method is taken as 15 .This method, is also known as Autocorrelation method, that fits as Auto Regressive (AR) model to the windowed input data. This block minimizes the forward prediction errors using the least squares sense. This formulation leads to the Yule-Walker equations, which the Levinson-Durbin recursion solves. Block outputs are always non-singular.

The input is a frame-based vector (column only). This input represents a frame of consecutive time samples from a single-channel signal. The block outputs a column vector containing the estimate of the power spectral density of the signal at $\mathrm{N}_{\text {fft }}$ equally spaced frequency points. The frequency points are in the range $\left[0, F_{s}\right)$, where $F_{s}$ is the sampling frequency of the signal[13]. The power spectral density obtained from Yule Walker method is shown in the Figure 11 .



Fig 11: Power Spectral Density from Yule Walker Method

The results obtained from BURG method and Yule Walker Method were shown in figure 12.

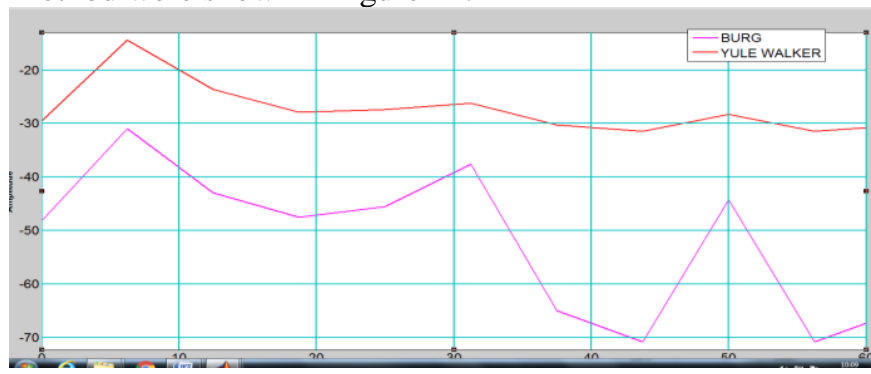

Fig 12: Power Spectral Densities Obtained From BURG and Yule Walker Method

The overall set up of estimation of power spectral density of EEG signal using SIMULINK is shown Fig 13.

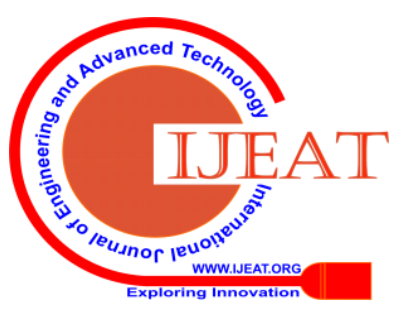



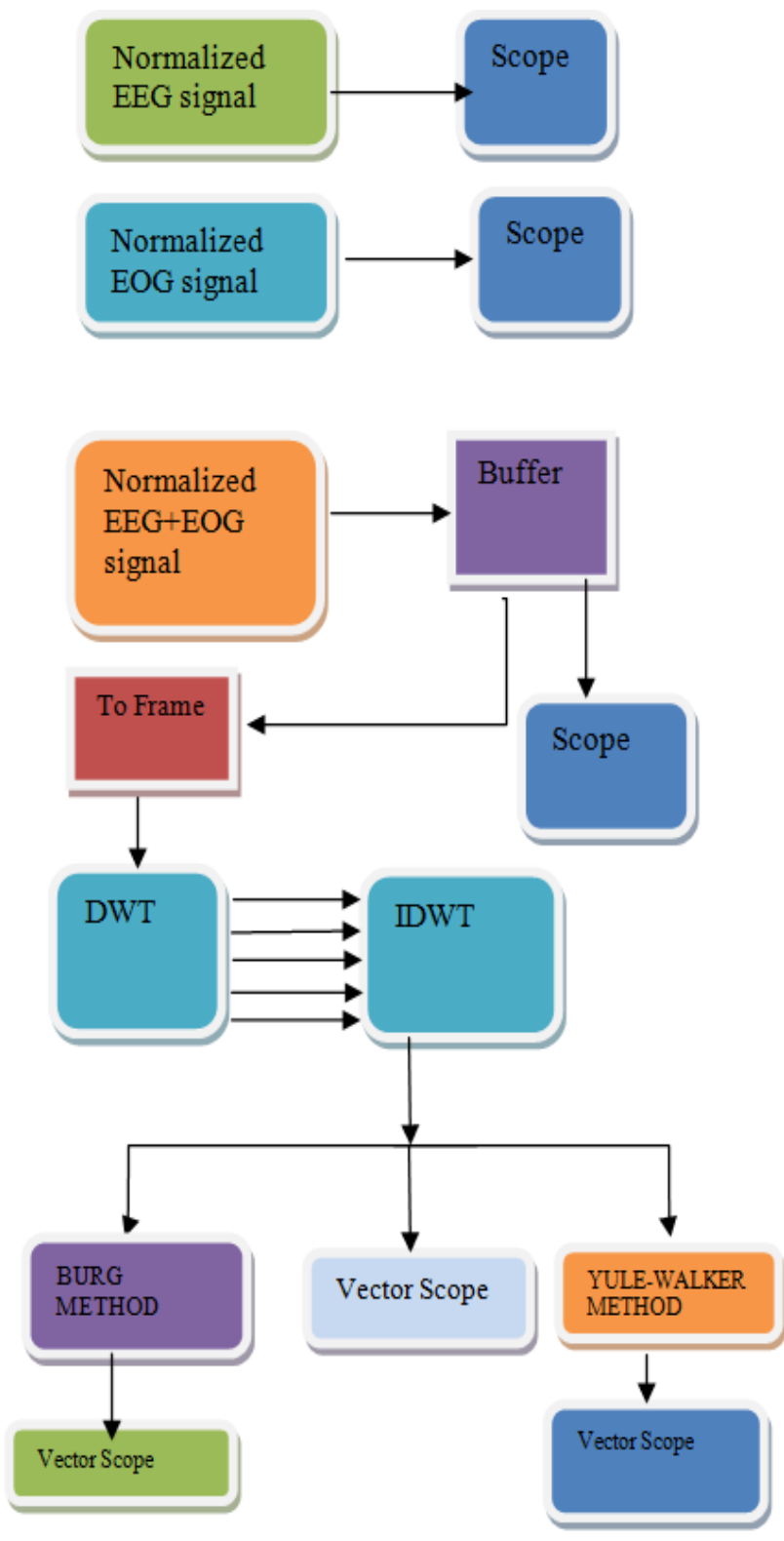

Fig 13: overall set up of estimation of power spectral density of EEG signal using SIMULINK

III RESULTS

The estimation of SNR of denoised signal using different wavelets was presented against method of implementation (MATLAB OR SIMULINK) in Table 1.

Table 1: SNR in dB using MATLAB and SIMULINK

\begin{tabular}{|c|c|c|}
\hline \multirow{2}{*}{$\begin{array}{l}\text { Name of } \\
\text { Wavelet }\end{array}$} & \multicolumn{2}{|c|}{ SNR IN dB } \\
\hline & MATLAB & SIMULINK \\
\hline DB4 & 5.625 & 11.8644 \\
\hline DB10 & 6.302 & 11.7018 \\
\hline Sym8 & 5.6658 & 11.5744 \\
\hline Haar & 5.5616 & 11.4650 \\
\hline
\end{tabular}

\section{CONCLUSION}

From the Table 1, it is observed that the signal denoising using SIMULINK is providing better results than MATLAB. From figure 12, it has been observed that the frequency components of the EEG signal are falling in the range of $0-12 \mathrm{~Hz}$ and $45 \mathrm{~Hz}-55 \mathrm{~Hz}$. The Burg method is providing better spectrum of EEG signal over Yule Walker method.

\section{REFERENCES}

1. ENiedermeyer and FHSilva "Electroencephalography: Basic principles, clinical applications and related fields" Lippincott, Williams \& Wilkins, 2004

2. MR Arab, AA Suratgar, VMM Hernandez, AR Ashtiani "Electroencephalogram Signals Processing for the Diagnosis of Petit mal and Grand mal Epilepsies Using an Artificial Neural Network' Journal of Applied Research and Technology, vol 8, pp. 120-129, 2010

3. GL Holmes, CT Lombroso. "Prognostic value of background patterns in the neonatal EEG" .J. Clin. Neurophysiol, Vol.10, pp. 323-352, 1993.

4. S Almubarak, PK Wong. "Long-Term Clinical Outcome of Neonatal EEG Findings J.Clin.Neurophysiol,vol.28, pp.185- 189, 2011.

5. ASM Muthanantha Murugavel, S Ramakrishnan.Tree Based Wavelet Transform and DAG SVM for Seizure Detection.Signal and Image Processing: An International Journal, vol.3, pp. 115-125, 2012

6. N.V. Thakor et al. "Multi resolution Wavelet Analysis of Evoked Potentials", IEEE Transactions on Biomedical Engineering, Vol. 40, No 11, pp. 1085-1093,November, 1993.

7. S. Ventakaramanan, P. Prabhat, S.R Choudhury, H.B Nemade, and J.S Sahambi,"Biomedical Instrumentation Based On Electrooculogram (EOG) Signal Processing And Application To A Hospital Alarm System", Indian Institute Of Technology (IIT) Gauhati, Proceedings of IEEE ICISEP, pp.535-539, 2000.

8. Schlogl A, Keinrath C, Zimmermann D et al.. A fully automated correction method of EOG artifacts in EEG recordings. Clinical Neurophysiology. Vol 118, pp98-104, 2007.

9. Jung T-P, Makeig S, Humphries C, Lee T-W, McKeown MJ,Iragui V and Sejnowski TJ. "Removing Electroencephalographic artifacts by blind source separation" .Psychophysiology. Vol.37, pp163 -178 2000.

10.Gratton G, Coles MG and Donchin E "A new method for off-line removal of oucular artifact. Electroencephalography" Clin.Neurophysiol vol. 55, pp484- 486, 1983

11. www.physionet.org

12.B. K. Kumar, K. V. S. V. R. Prasad and D. Alekhya, "Performance comparison of various thresholding techniques on the removal of ocular artifacts in The EEG signals," International Conference on Inventive Computation Technologies (ICICT), Coimbatore, 2016.

13. www. Mathworks.com

\section{AUTHORS PROFILE}

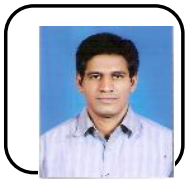

B.Krishna Kumar holds $\mathrm{PhD}$ degree in Electronics and Communication Engineering from Jawaharlal Nehru Technological University, Hyerabad. He also has M.Tech degree in Digital Systems and Computer Electronics from JNTU, Hyderabad and B.Tech degree in Electronics and Communication Engineering from S.V.University, Tirupathi. He has more than 15 publications to his credit. He has written a book on Estimation of statistical parameters of EEG signals using wavelets. $\mathrm{He}$ is a member of INSTITUTE OF RESEARCH ENGINEERS AND DOCTORS and also International Association of Engineers. His fields of interest are biomedical signal processing and image processing. 